\title{
Delivering Interconception Care During Well-Child Visits: An IMPLICIT Network Study
}

\author{
Sukanya Srinivasan, MD, MPH, Lisa Schlar, MD, Stephanie E. Rosener, MD, IBCLC, \\ Daniel J. Frayne, MD, Scott G. Hartman, MD, Michael A. Horst, PhD, MPHS, MS, \\ Jessica L. Brubach, MPA, and Stephen Ratcliffe, MD, MSPH
}

Background: Preterm birth, birth defects, and unintended pregnancy are major sources of infant and maternal morbidity, mortality, and associated resource use in American health care. Interconception Care (ICC) is recommended as a strategy to improve birth outcomes by modifying maternal risks between pregnancies, but no established model currently exists. The Interventions to Minimize Preterm and Low Birth Weight Infants through Continuous Improvement Techniques (IMPLICIT) Network developed and implemented a unique approach to ICC by assessing mothers during their baby's well-child visits (WCVs) up to 24 months.

Methods: Mothers who accompanied their children to WCVs at eleven eastern US family medicine residency programs underwent screening for four risk factors (tobacco use, depression risk, contraception use to avoid unintended pregnancy and prolong interpregnancy interval, and use of a multivitamin with folic acid). Positive screens in women were addressed through brief interventions or referrals to treatment.

Results: Mothers accompanied their babies to $92.7 \%$ of WCVs. At more than half of WCVs (69.1\%), mothers were screened for presence of ICC behavioral risks, although significant practice variation existed. Risk factors were identified at significant rates (tobacco use, 16.2\%; depression risk, 8.1\%; lack of contraception use, $28.2 \%$; lack of multivitamin use, $45.4 \%$ ). Women screened positive for 1 or more ICC risk factor at $64.6 \%$ of WCVs. Rates of documented interventions for women who screened positive were also substantial (tobacco use, $\mathbf{8 0 . 0 \%}$; depression risk, $92.8 \%$; lack of contraception use, $76.0 \%$; lack of multivitamin use, $\mathbf{5 8 . 2 \%}$ ).

Conclusion: WCVs provide a reliable point of contact with mothers and a unique opportunity to assess and address behavioral risks for future poor birth outcomes. (J Am Board Fam Med 2018;31: 201-210.)

Keywords: Interconception Care, Maternal Assessment, Preterm Birth, Unplanned Pregnancy, Well-Child Visits

The United States ranks 44th in the world in infant mortality and 49th in maternal mortality. ${ }^{1}$ Despite a number of strategies intended to improve pregnancy care, the US infant mortality rate has dropped only slightly and the maternal mortality

This article was externally peer reviewed.

Submitted 10 June 2017; revised 7 December 2017; accepted 10 December 2017.

From the University of Pittsburgh Medical Center (UPMC) St. Margaret Family Medicine Residency, Pittsburgh, PA (SS); UPMC Shadyside Family Medicine Residency, Pittsburgh (LS); Middlesex Hospital Family Medicine Residency Program, Middletown, CT (SER); MAHEC Family Health Centers, Asheville, NC (DJF); Department of Family Medicine, University of Rochester, Rochester, NY (SGH); Lancaster General Research Institute, Lancaster, rate is rising. ${ }^{2}$ Almost half of pregnancies in the US are unintended (mistimed or unwanted) ${ }^{3}$ and only $22.8 \%$ percent of US women report receiving pre-

PA (MAH); IMPLICIT Network and UPMC McKeesport, Shadyside and St. Margaret Family Health Centers, Pittsburgh (JLB); Lancaster General Family Medicine Residency, Lancaster (SR).

Funding: Funding support for the development of this model of care was provided by National March of Dimes; Pennsylvania, New York and North Carolina March of Dimes; Pennsylvania Department of Health; Pennsylvania Chapter of the American Academy of Pediatrics; Shadyside Hospital Foundation, Pittsburgh, PA; St. Margaret Hospital Foundation, Pittsburgh, PA.

Conflict of interest: none declared.

Corresponding author: Sukanya Srinivasan, MD, 7175 Saltsburg Road, Pittsburgh, PA 15235 (E-mail: srinivasans@upmc. edu). 
conception care. ${ }^{4}$ By the time a woman begins prenatal care, it is often too late to modify many of the risk factors associated with poor birth outcomes. ${ }^{5}$

Interconception care (ICC) is defined as care for women of childbearing age between pregnancies (from the end of one pregnancy to conception of the next $)^{6}$ and includes medical and psychological interventions to modify risk factors in women to improve future birth outcomes. ${ }^{7}$ In 2006, the Centers for Disease Control and Prevention Work Group and Select Panel on Preconception Care recommended risk assessment and intervention in the interconception period, especially for women with previous adverse outcomes. ${ }^{8}$

Although broadly advocated by national groups, ICC has not been widely implemented. ${ }^{8,9}$ Inadequate knowledge among clinicians, lack of an established model, and multiple barriers to care prevent widespread delivery of effective interventions. ${ }^{5,10}$ After the birth of a child, many women who received regular prenatal care stop seeing providers for their own health care or return to a pattern of fragmented care. ${ }^{11-16}$ During this time, women often revert to high-risk behaviors such as smoking and substance abuse. ${ }^{17-19}$ In addition, the maternal and family focus shifts from the woman to the infant, ignoring the health care needs of the mother. ${ }^{16,20}$

Continuity of care with the same primary care clinician or practice over time is associated with improved outcomes, including increased use of preventive services, better adherence to clinician recommendations, and lower total costs, although it has not been well studied in the context of maternal and child care delivery. ${ }^{8,21-23}$ Mothers regularly attend their child's health care visits even if they do not seek care for themselves between pregnancies. $^{20,24}$ Given that children typically attend 8 to 10 well-child visits in the first 2 years of life, these visits offer a consistent point of maternal contact and opportunity for assessment. Furthermore, prior studies demonstrate that most mothers accept inquiry about their own health behaviors and referral for services at visits with their child's pediatric care provider. ${ }^{24-31}$

The Interventions to Minimize Preterm and Low Birth Weight Infants through Continuous Improvement Techniques (IMPLICIT) Network, a family medicine maternal child health learning collaborative of the Family Medicine Education Consortium, created a model of ICC that addresses barriers to care by screening women during wellchild visits (WCVs). The IMPLICIT Network develops, implements, evaluates, and optimizes new and existing models of care focused on improving birth outcomes and the health of women, infants, and families (Figure 1). ${ }^{32}$ Previous Network efforts include development of continuous quality improvement processes to improve prenatal care, validation of a 2-step depression screening process during pregnancy, and assessment of current interconception practices by family physicians at WCVs. ${ }^{31,33,34}$ Since 2012, the Network has focused on developing and implementing an ICC model to address specific maternal risk factors for poor subsequent birth outcomes.

\section{IMPLICIT Network ICC Model}

Other models for ICC rely on maternal office visits but this approach may not reach women who are at highest risk for adverse birth outcomes such as low income, younger, or minority women. The IMPLICIT Network's model (IMPLICIT ICC) targets all women when they accompany their children to well-child visits, enhancing access to a health care provider especially for women who may not otherwise seek care. The IMPLICIT ICC model incorporates 2 key concepts: 1) Screening and interventions are brief and performable within the context of a primary care well-child visit; 2) targeted screenings and interventions have strong evidence (SORT A or B) for improving future birth outcomes. Although there are many maternal behaviors and conditions that could be included in a broader approach to interconception care, the IMPLICIT ICC model is designed as a brief screening and intervention strategy. Therefore, we chose to focus on four intervention areas with strong evidence for improving birth outcomes also readily delivered in the setting of a WCV. ${ }^{8,36-40}$ (Figure 2)

1. Tobacco use: Smoking cigarettes during pregnancy has been clearly linked to poor birth outcomes including low birth weight and prematurity. Cessation literature consistently demonstrates that approximately $50 \%$ of prenatal tobacco quitters have relapsed by 6 months postpartum (i.e., during the interconception time period). Use of structured 
Figure 1. Map of IMPLICIT Network Family Medicine Practices.

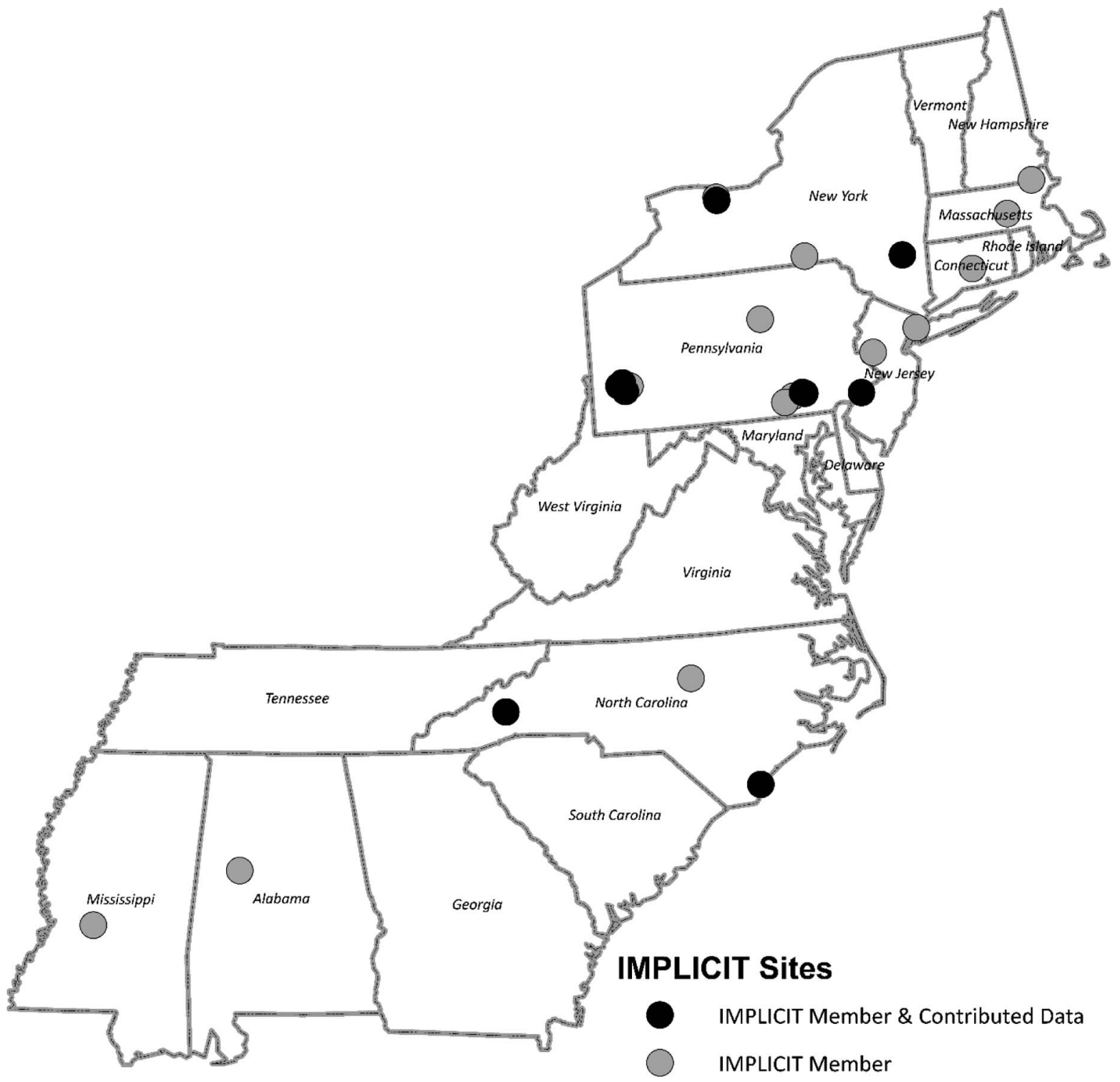

tobacco use questions and brief interventions has been validated to improve cessation rates. ${ }^{18,19,31,34}$

2. Depression: Maternal depression has significant negative impacts for both mother and children, during pregnancy and afterward. Preconception and interconception screening and assessment of mothers for depression risk is recommended by the American Academy of Pediatrics as a routine part of pediatric care in the first year of life. ${ }^{24-28,33,34}$

3. Family planning: Unintended pregnancies have been associated with detrimental maternal behaviors during pregnancy and an increased

risk of low birth weight and preterm outcomes. Birth spacing beyond 18 months by promoting adherence to contraception is a key component of the model. ${ }^{29,31-34}$

4. Use of multivitamin with folic acid (MVI): Only $24 \%$ of nonpregnant US women ages 15 to 44 years consume the recommended intake of folic acid even though consistent intake of folic acid is an established recommendation. Recommending and providing multivitamin/folic acid universally for all women in the preconception/interconception period is another critical goal of IMPLICIT interconception care. ., $^{10,31,34,36}$ 
Figure 2. IMPLICIT Network Interconception Care (ICC) Model work flow. QI, quality improvement; PHQ-9, 9question Patient Health Questionnaire; LARC, long-acting reversible contraception.

\section{IMPLICIT ICC Model}

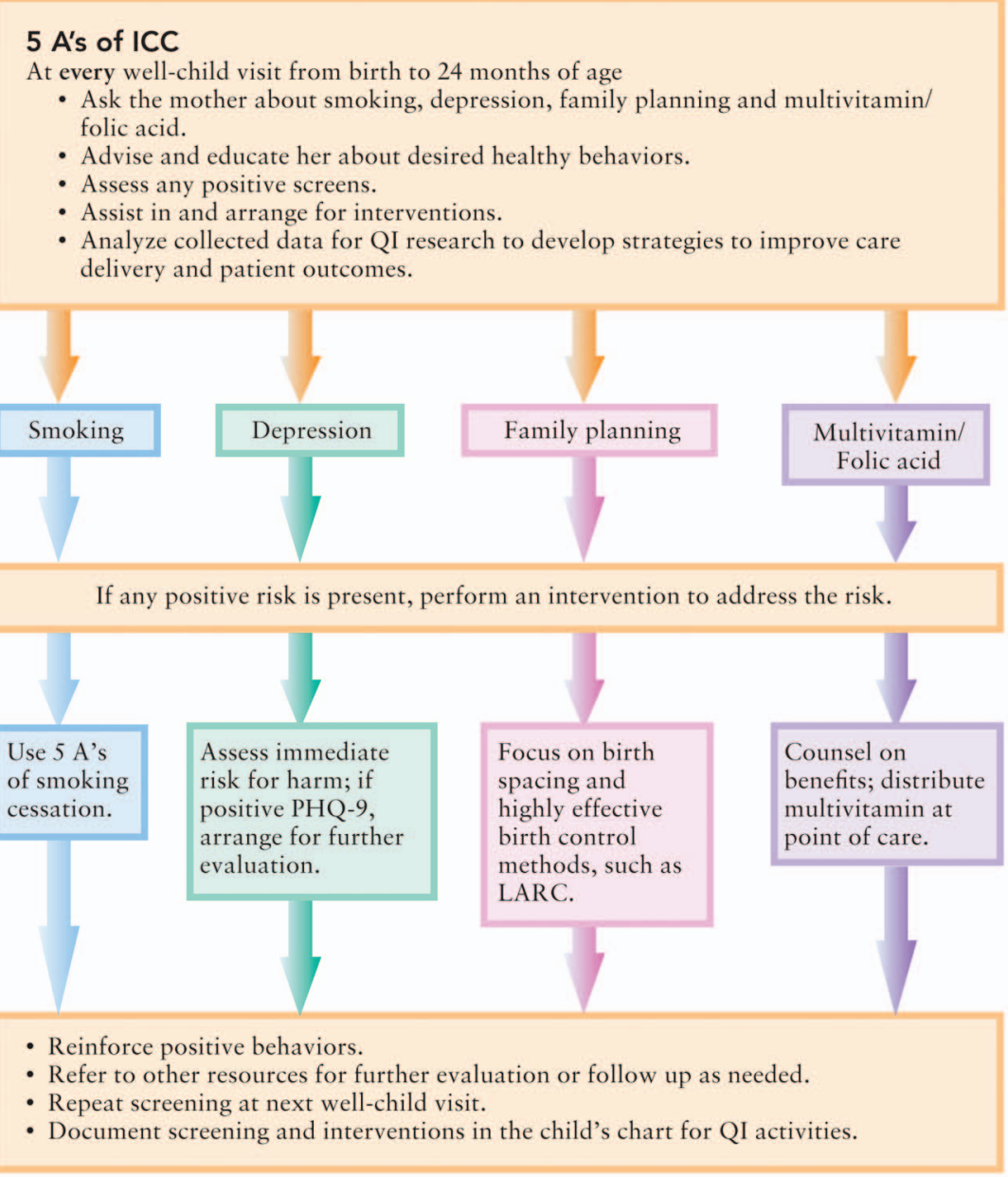

The IMPLICIT ICC model adapts the familiar 5 A's of behavioral change theory, a model recommended for smoking cessation for more than 20 years by the National Cancer Institute ${ }^{35}$ to these focused behavioral risks.

\section{Methods}

\section{Study Design}

Family medicine clinical practices affiliated with eleven eastern US family medicine residency programs participating in the IMPLICIT Network implemented the IMPLICIT ICC model as a quality improvement initiative. Two sites are community-based residency programs and 9 are community-based, university-affiliated programs. Practice settings varied from urban to suburban to rural.
Subjects included all mothers 13 years and older who presented with their children for newborn to 24-month WCVs. This interval was chosen because of the risk for poor birth outcomes associated with a subsequent pregnancy occurring within this time period. Implementation occurred at different times across practice sites with standardization of IMPLICIT ICC measures and processes completed on January 31, 2015. This study evaluates IMPLICIT ICC delivery to mothers at WCVs from February 1, 2015 until April 4, 2017. The primary objective is to describe the feasibility of implementing this model during WCVs by characterizing maternal attendance at WCVs and evaluating the frequency of maternal risk factors identified and addressed. All participating sites secured 
Institutional Review Board approval before project implementation.

\section{Screening Strategies}

Screening practices varied across the participating health centers and included both verbal and written strategies for assessing maternal behavioral risks at WCVs (Appendix A). Self-reported maternal demographics were obtained at their child's initial visit to the practice or collected through chart review after ICC screenings were completed (Appendix B). Clinicians screened mothers at well-child visits from 2 to 24 months. Mothers received screening and advice regardless of whether or not she received primary care from the same provider or practice. Clinicians recorded maternal responses to screening for the 4 risk factors directly in the child's health record since this information pertains to the wellbeing of the child and family, and so that responses would be readily available at subsequent visits for review.

When screening for tobacco use, clinicians or office staff asked mothers about smoking status and documented whether interventions were offered to encourage smoking cessation. Mothers were screened for depression using a 2-step strategy: administration of either the 2-question Patient Health Questionnaire (PHQ-2) or the 2-item Depression Screen ${ }^{41,42}$ was subsequently followed by the 9-question Patient Health Questionnaire (PHQ-9) for any mothers who responded positively to the initial screen. Any positive PHQ-2 or 2-item Depression Screen prompted an immediate safety assessment for postpartum psychosis and possible suicidality and documentation of appropriate triage of this high-risk situation. Clinicians documented whether an intervention was offered to women at significant risk for depression (PHQ-9 score $\geq 10$ ). Clinicians assessed pregnancy status, intent, and current method of contraception along with counseling and interventions. Use of long-acting reversible contraception (LARC) was specifically noted. Finally, providers assessed maternal use of multivitamin with folic acid and recommendations and interventions were documented.

A variety of approaches based on availability of resources at each site were utilized to address identified maternal risk factors including promoting healthy behaviors, providing prescriptions, and referrals for additional services. A variety of services were available to the participating clinicians on site including case management, social workers, com- munity health workers, substance abuse counselors, and office-based pharmacists. Each family medicine practice offered patients access to mental health counseling, with 6 of the 11 sites reporting availability of colocated, integrated behavioral health models. Clinicians utilized a standardized documentation format to ensure uniformity of recorded maternal responses. On a case-by-case basis, providers may have chosen to extend their interaction with the family and offer a visit concurrently for the mother, especially if the risk identified warranted immediate attention. De-identified data at each practice were either extracted from structured data fields in the electronic record or abstracted from article charts and entered into the Network's data management system, Research Electronic Data Capture (REDCap) ${ }^{43}$, hosted at Lancaster General Hospital.

\section{Data Analysis}

Descriptive statistics were used to characterize the Network's maternal sample, to evaluate the frequency of maternal ICC screening in each of the target risk-factor areas, and to assess the frequency of interventions for identified maternal risks at WCVs. Maternal screening rate was defined as the ratio of the number of visits where part or all the ICC questions were asked to the number of all possible WCVs (includes visits where the mother was present, was not present, or presence could not be verified), with response frequencies expressed as percentages. Each target risk factor was linked to discreet list of choices for intervention if screening was positive. Statistical analyses were performed using Stata version 14.1 (Stata Corporation, College Station, TX) and R version 3.3.1 (R Foundation for Statistical Computing, Vienna, Austria).

\section{Results}

Data collected from the mothers of 5927 individual children across $17,630 \mathrm{WCVs}$ are represented in the aggregate data set. The number of WCVs per site ranged from 33 to 5417 and the number of mothers ranged from 27 to 1478 (Appendix C). Sites with low numbers of currently enrolled mother child dyads were included to demonstrate the acceptance of the model. Demographic features of mothers surveyed are summarized based on data collected from approximately half of mothers accompanying their children to WCVs (Table 1). Of women who completed the demographic 
Table 1. Maternal Characteristics at Participating Sites

\begin{tabular}{lccc}
\hline Demographic & Total No. of Mothers & Average Rate (\%) & Range Across Sites (\%) \\
\hline Medical assistance & 2788 & 77.5 & 55.3 to 91.7 \\
Nonwhite race & 3745 & 70.5 & 36.0 to 100.0 \\
Hispanic & 2694 & 27.7 & 2.3 to 51.3 \\
Maternal education* & & 38.2 & 4.7 to 67.6 \\
$\quad$ Less than high school degree or equivalent & 1098 & 35.7 & 15.7 to 56.9 \\
$\quad$ High school graduate or equivalent & 1026 & & 0.3 \\
Maternal age (years) & & 8.3 & 0.3 \\
$\quad<15$ & 8 & 31.4 & 6.2 to 10.2 \\
15 to 19 & 251 & 945 & 23.7 to 40.0 \\
20 to 24 & & & \\
\hline
\end{tabular}

*Not collected at every site.

form, $70.5 \%$ self identified as nonwhite race and $77.5 \%$ reported coverage by government-sponsored health insurance. Across participating sites, mothers self identified as patients of the practice at an average rate of $83.5 \%$. Of the WCVs where maternal presence or absence could be verified $(\mathrm{n}=$ $12,171)$, mothers accompanied their children to $92.7 \%$ of visits. The frequency of maternal screening at participating sites ranged widely from $59.7 \%$ to $98.4 \%$, with women receiving ICC screening at an average rate of $69.1 \%$ of WCVs across all study sites.

The frequency of maternal risk identification and intervention frequency for each risk factor was calculated (Figure 3). The percentage of WCVs where maternal behavioral risks were identified varied across sites and across the four risk factors. Clinicians identified tobacco use in $16.2 \%$ of visits, depression in $8.1 \%$ of visits, lack of contraception use in $28.2 \%$ of visits, and lack of multivitamin use by women in $45.4 \%$ of WCVs. Across all sites, $63.6 \%$ of women screened had at least one risk factor identified.

Clinicians delivered interventions for tobacco use at $80.0 \%$ of the visits where the mother was currently smoking. Women at risk for depression received an intervention $92.8 \%$ of the time. At 49 WCVs, women who screened positive with the PHQ-2 also reported to have thoughts of self harm $(4.3 \%)$ and at 34 of those WCVs, documentation reflects maternal assessment for safety and appropriate triage to ensure no immediate threat existed. Of women identified as not using contraception at the time of their child's WCV, $76.0 \%$ received an intervention to promote adequate birth spacing. Contraception use ranged from $58.3 \%$ to $87.6 \%$ across all the sites with $22.6 \%$ of women reporting use of LARC methods. Finally, in $58.2 \%$ of instances where mothers reported inadequate multivitamin use, an intervention was documented.

\section{Discussion}

This 26-month study of maternal attendance and provider screening rates at family medicine clinical practices that implemented the IMPLICIT interconception care model demonstrates the feasibility of using WCVs to screen for maternal behavioral risks. Mothers accompanied their children to the overwhelming majority of WCVs under two years of age. Across participating sites, women received partial or complete ICC screening (based on the IMPLICIT ICC model) from their child's health care provider at more than two thirds of all WCVs. The consistency of maternal attendance and provider screening at these visits suggests that wellchild office visits are a viable and accessible point of contact with women in the critical time between pregnancies.

Another outcome of this study is the demonstration of the feasibility of delivering interconception care through the IMPLICIT ICC model. Nearly 6000 unique mothers were screened across the eleven sites while accompanying their children to well-child visits. Approximately two thirds of the women had modifiable risk factors for adverse pregnancy outcomes and most women obtained some type of intervention to modify these behavioral risks while their child received well-child care. Since services such as case management, behavioral health, substance abuse counselors, social workers, or pharmacists may have been available on site to 
Figure 3. Network versus individual site rates of identified risks and documented interventions at well-child visits (February 1, 2015 to April 4, 2017). ICC, interconception care; WCV, well-child visits.

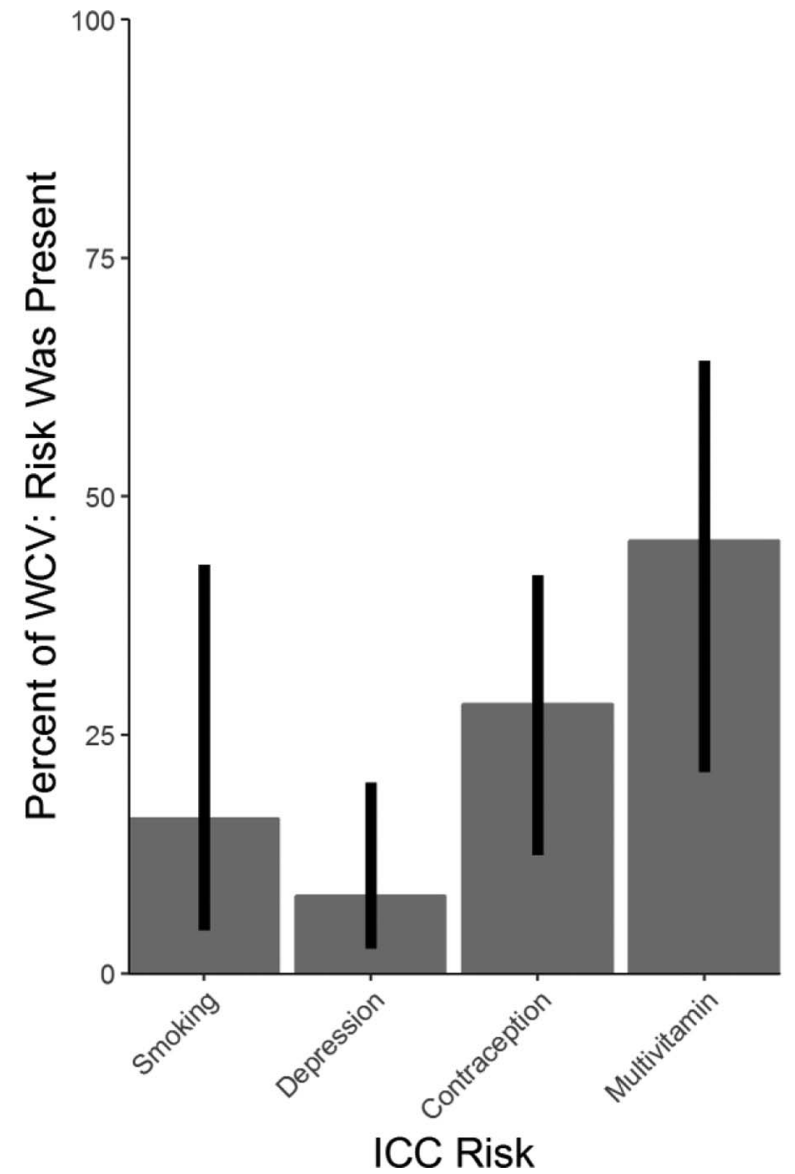

the participating clinicians, risks could be directly addressed in the context of the child's medical home. Furthermore, some interventions could even be delivered (such as provision of multivitamins) even when women identified an alternative clinician or practice as their source of primary care, offering an additional way to facilitate care for women who otherwise may not attend to their own health concerns.

This study has several limitations. First, the ability to verify maternal presence at WCVs was limited for comparison among participating sites because of inconsistent documentation of who accompanied the child to the visit. Some sites did not specifically document whether the child's mother was present at each WCV, restricting our ability to measure the missed opportunities for screening and intervention (e.g. when the mother was present but ICC screening was not completed). Second, although a standardized set of questions were used for maternal screening, variations in workflow among sites

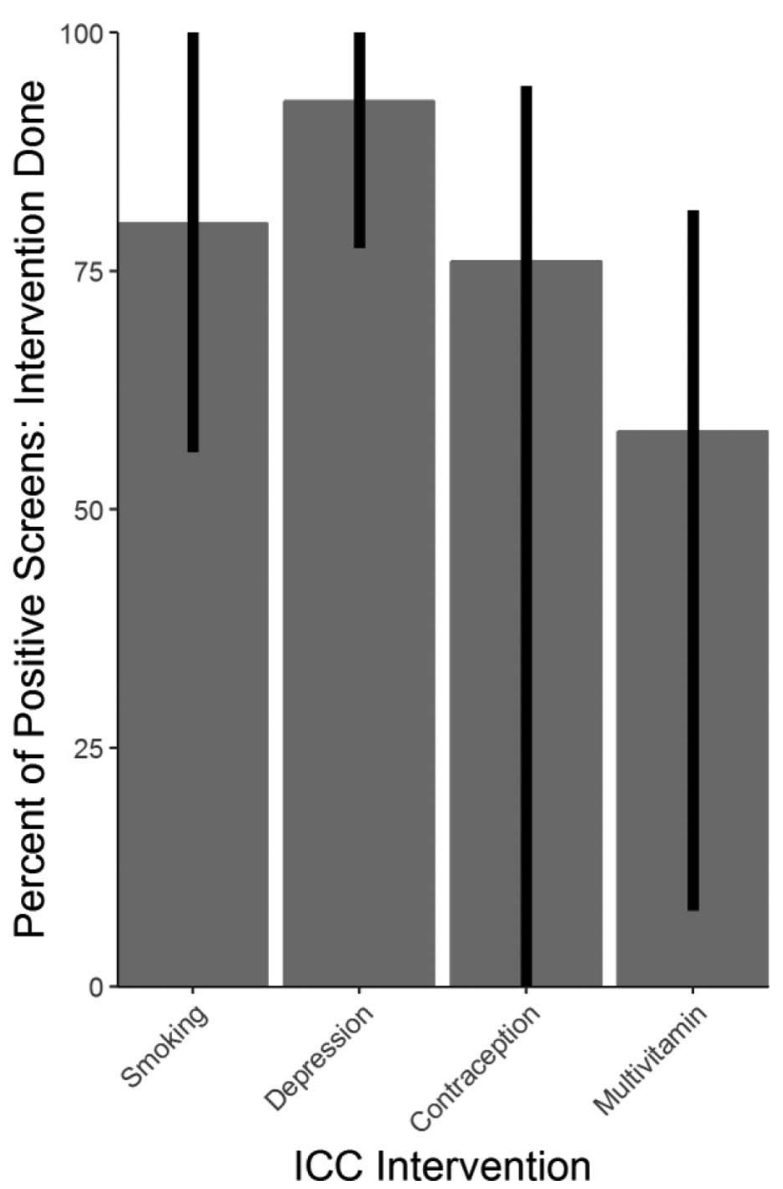

during implementation contributed to the wide range of identified maternal risk factors and intervention rates. Rates of ICC maternal screening varied from $59.7 \%$ to $98.4 \%$ and some of this variation may be a result of the way responses were documented. For example, at some sites, staff members documented mothers' smoking status separately from clinician screening, so that even if the visit provider did not formally ask the remainder of the screening questions, responses for smoking were recorded.

A third limitation is the difference in documentation methods among participating sites. Some sites captured data from structured fields in the child's electronic medical record while others performed manual data abstraction from the child's record, which could have led to interpretation or transcription errors. A final limitation is the broad definition of intervention that the IMPLICIT ICC model adopted, placing the primary emphasis on documenting the providers attempts to address the 
identified risk. Given the compactness of the model, the ability to report specific patient care details was not available, making it difficult to validate the specific outcomes from ICC screening.

Despite these limitations, the IMPLICIT ICC model holds promise as a brief maternal screening and intervention strategy for family medicine clinical practices as well as other primary care settings, such as community health centers and pediatric practices. This model can close gaps in care by first identifying risks in a population of women whose needs might otherwise go unaddressed. Engaging these women in their own care and connecting them with resources using ICC screening at WCVs as an entry point offers great potential benefit for not only mothers but also their babies and families. Because of the model's flexibility, sites can readily customize implementation by incorporating the questions directly into existing workflows and using local service providers already working in maternal-child health.

Although practices with colocated maternity, children's and adult primary care may be able to access the maternal chart for screening and documentation or even schedule maternal visit, the IMPLICIT ICC model was developed for use in busy primary care practices or settings in which all resources and care models are often not colocated.

Based on the findings of this study and the clinical experiences of participating sites with the IMPLICIT ICC model, several key recommendations can be offered to clinical practices seeking to implement this model for interconception care. Practices should develop standardized screening protocols, tools for point-of-care intervention for women who screen positive in any of the four key behavior risk areas, such as patient education materials and clinical management algorithms, and linkages with local community agencies so they may refer women needing additional resources not offered on site, such as depression care or contraception access. Practices should also strive to use quality improvement techniques to improve both screening and intervention rates. Practices that serve populations with limited resources such as uninsured, undocumented, or immigrant communities would gain particular benefit from implementing IMPLICIT ICC as a way to reach women not seeking care. Based on their particular population's needs, clinical practices might consider expanding the IMPLICIT ICC model to include ad- ditional risk factors for poor birth outcomes, such as domestic violence, food insecurity, obesity, or substance abuse. However, adding additional screening targets could limit the feasibility of screening and intervention in the context of the well-child visit. The use of the WCV is one of many strategies that providers may use to deliver the full breadth of comprehensive interconception care that women should receive. Future effectiveness studies are needed to assess rates of prematurity and other birth outcomes in populations who received interconception care through the IMPLICIT ICC model, especially at sites who have implemented the model for several years, to inform the growing literature on preconception care.

The authors would like to thank all the participating members of the IMPLICIT Network for their dedication and contribution to the project's success. Special thanks to the Family Medicine Education Consortium (FMEC).

To see this article online, please go to: bttp://jabfm.org/content/ 31/2/201.full.

\section{References}

1. World Bank Mortality rate, infant (per 1,000 live births). Retrieved from http://data.worldbank.org/ indicator/SP.DYN.IMRT.IN.

2. National Center for Health Statistics (2013). Infant mortality rates: United States, 2003 to 2013. Available from: http://www.marchofdimes.org/Peristats/ ViewSubtopic.aspx? $\mathrm{reg}=99 \&$ top $=6 \&$ stop $=91 \&$ lev $=1$ \&slev $=1 \&$ obj $=1$.

3. Finer LB, Zolna MR. Declines in unintended pregnancy in the United States, 2008-2011. N Engl J Med 2016;374:843-52.

4. Robbins CL, Zapata LB, Farr SL, et al. Core state preconception health indicators-Pregnancy risk assessment monitoring system and behavioral risk factor surveillance system, 2009. MMWR Surveill Summ 2014;63:1-62.

5. Atrash H, Jack BW, Johnson K. Preconception care: A 2008 update. Curr Opin Obstet Gynecol 2008;20: 581-9.

6. Howson CP, Kinney MV, Lawn JE, eds. March of Dimes, PMNCH, Save the Children, WHO. Born too soon: The global action report on preterm birth. Geneva, Switzerland: World Health Organization; 2012.

7. Yonekura ML, French J, Johnson RE, McGregor J, Reyes C. LA Best Babies Network. Perinatal scorecard (Report). Los Angeles, CA: LA Best Babies Network; 2009. Available from: http://labestbabies. org/publications/la-best-babies-network-perinatalscorecard. 
8. Johnson K, Posner S, Bierman J, et al. A report of the CDC/ATSDR Preconception Care Work Group and the Select Panel on Preconception Care. Recommendations to improve preconception health and health care-United States. MMWR Recomm Rep 2006;55:1-23.

9. Biermann J, Dunlop AL, Brady C, Dubin C, Brann A Jr. Promising practices in preconception care for women at risk for poor health and pregnancy outcomes. Matern Child Health J. 2006;10(5 Suppl): S21-8.

10. Jack BW, Culpepper L. Preconception care. Risk reduction and health promotion in preparation for pregnancy. JAMA 1990;264:1147-9.

11. DiBari JN, Yu SM, Chao SM, Lu MC. Use of postpartum care: Predictors and barriers. J Pregnancy 2014;2014:530769.

12. Jack BW, Atrash H, Bickmore T, Johnson K. The future of preconception care: A clinical perspective. Womens Health Issues 2008;18(6 Suppl): S19-25.

13. Liberto T. Screening for depression and help-seeking in postpartum depression during well-baby pediatric visits: An integrated review. J Pediatr Health Care 2012;26:109-17.

14. McGarry J, Kim H, Sheng X, EggerM, Baksh L. Postpartum depression and help-seeking behavior. J Midwifery Women's Health 2009;54:50-6.

15. Moos MK. From concept to practice: Reflections on the preconception health agenda. J Womens Health (Larchmt) 2010;19:561-7.

16. Salganicoff A, Ranji U, Wyn R. Women and their health care providers. In: Women and Health Care: A National Profile-Report. The Henry J. Kaiser Family Foundation. June 29, 2005. Available from: http://kff.org/disparitiespolicy/report/women-andhealth-care-a-national-profile.

17. US Department of Health and Human Services. Women's health USA. Rockville, MD: US Department of Health and Human Services, Health Resources and Services Administration; 2005.

18. Substance abuse in pregnancy. ACOG Technical Bulletin Number 195-July 1994 (replaces No. 96, September 1986). Int J Gynaecol Obstet 1994;47: 73-80.

19. Fang WL, Goldstein AO, Butzen AY, et al. Smoking cessation in pregnancy: A review of postpartum relapse prevention strategies. J Am Board Fam Pract 2004; 17:264-75.

20. Bloom B, Cohen RA, Freeman G. National Center for Health Statistics Summary health statistics for U.S. children: National Health Interview Survey, 2009. Vital Health Stat 10 2010;Dec(247): 1-82.

21. Moos MK. Preconceptional health promotion: Opportunities abound. Matern Child Health J 2002;6: 71-3.

22. Weisman CS. Changing definitions of women's health: Implications for health care and policy. Matern Child Health J 1997;1:179-89.

23. Clancy CM, Massion CT. American women's health care. A patchwork quilt with gaps. JAMA 1992;268: 1918-20.

24. Gjerdingen D, Crow S, McGovern P, Miner M, Center B. Postpartum depression screening at wellchild visits: Validity of a 2-question screen and the PHQ-9. Ann Fam Med 2009;7:63-70.

25. Feinberg E, Smith MV, Morales MJ, Claussen AH, Smith DC, Perou R. Improving women's health during internatal periods: Developing an evidencedbased approach to addressing maternal depression in pediatric settings. J Womens Health (Larchmt) 2006;15:692-703.

26. Freeman MP, Wright R, Watchman M, et al. Postpartum depression assessments at well-baby visits: Screening feasibility, prevalence, and risk factors. J Womens Health (Larchmt) 2005;14:929-35.

27. Heneghan AM, Mercer M, DeLeone NL. Will mothers discuss parenting stress and depressive symptoms with their child's pediatrician? Pediatrics 2004;113(3 Pt 1):460.

28. Kahn RS, Wise PH, Finkelstein JA, Bernstein HH, Lowe JA, Homer CJ. The scope of unmet maternal health needs in pediatric settings. Pediatrics 1999; 103:576

29. Trussell J, Henry N, Hassan F, Prezioso A, Law A, Filonenko A. Burden of unintended pregnancy in the United States: Potential savings with increased use of long-acting reversible contraception. Contraception 2013;87:154-61.

30. Wilson CR, Harris SK, Sherritt L, et al. Parental alcohol screening in pediatric practices. Pediatrics 2008;122(5):e1022-9.

31. Rosener SE, Barr WB, Frayne DJ, Barash JH, Gross ME, Bennett IM. Interconception care for mothers during well-child visits with family physicians: An IMPLICIT Network Study. Ann Fam Med 2016;14: 350-5.

32. IMPLICIT Network-An FMEC Collaborative. 2012. Available from: www.fmec.implicit.net. Accessed on May 11, 2017.

33. Bennett IM, Coco A, Coyne JC, et al. Efficiency of a two-item pre-screen to reduce the burden of depression screening in pregnancy and postpartum: An IMPLICIT Network study. J Am Board Fam Med 2008;21:317-25.

34. Bennett IM, Coco A, Anderson J, et al. Improving maternal care with a continuous quality improvement strategy: A report from the Interventions to Minimize Preterm and Low Birth Weight Infants through Continuous Improvement Techniques (IMPLICIT)Network. J Am Board Fam Med 2009;22:380-6.

35. Glynn TJ, Manley MW. How to help your patients stop smoking: A National Cancer Institute manual for physicians. Bethesda, MD: National Cancer Institute; 1997. 
36. Bukowski R, Malone FD, Porter FT, et al. Preconceptional folate supplementation and the risk of spontaneous preterm birth: A cohort study. PLoS Med 2009;6(5):e1000061.

37. Conde-Agudelo A, Rosas-Bermúdez A, KafuryGoeta AC. Birth spacing and risk of adverse perinatal outcomes: A meta-analysis. JAMA 2006;295: 1809-23.

38. Frieder A, Dunlop AL, Culpepper L, Bernstein PS. The clinical content of preconception care: Women with psychiatric conditions. Am J Obstet Gynecol 2008;199(6 Suppl 2):S328-32.

39. Wilson RD, Johnson JA, Wyatt P, et al.; Genetics Committee of the Society of Obstetricians and Gynaecologists of Canada and The Motherrisk Program. Pre-conceptional vitamin/folic acid supplementation 2007: The use of folic acid in combination with a multivitamin supplement for the prevention of neural tube defects and other congenital anomalies. [Published correction appears in J Obstet Gynaecol Can 2008;30:193] J Obstet Gynaecol Can 2007;29:1003-26.

40. Ebbert JO, Jacobson RM. Reducing childhood tobacco smoke exposure. JAMA 2016;315:2610-1.

41. Kroenke K, Spitzer RL, Williams JB. The PHQ-9: Validity of a brief depression severity measure. J Gen Intern Med 2001;16:606-13.

42. Kroenke K, Spitzer RL, Williams JB. The Patient Health Questionnaire-2: Validity of a two-item depression screener. Med Care 2003;41:1284-92.

43. Harris PA, Taylor R, Thielke R, Payne J, Gonzalez N, Conde JG. Research electronic data capture (REDCap) - A metadata-driven methodology and workflow process for providing translational research informatics support. J Biomed Inform 2009; 42:377-81. 